Contribution to RNB4, Fourth International Conference on Radioactive Nuclear Beams, Omiya, Japan, June 4-7 1996

\title{
How large are the halos of light nuclei?
}

\author{
J.A. Tostevin* and J.S. Al-Khalilia
}

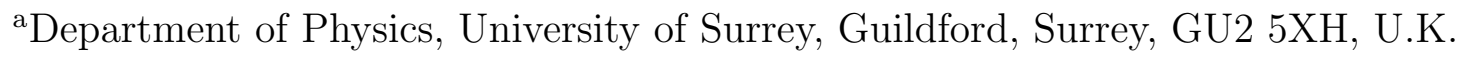

We present quantitative reaction cross section calculations for halo nuclei at high energy which retain the essential few-body correlations in such structures. Inclusion of these correlations leads to larger deduced halo radii. For three-body $(n+n+$ core $)$ projectiles, cross section measurements determine the rms hyperradius of the system and, given the core nucleus size, the projectile matter radius. The importance of finite range effects are estimated in the case of the lightest two-neutron-halo system ${ }^{6} \mathrm{He}$. Outstanding uncertainties are discussed.

\section{Introduction}

Interaction cross section (transmission) measurements at energies of several hundred $\mathrm{MeV} / \mathrm{A}$ have been used to deduce the radial extent of short lived exotic nuclei from fragmentation reactions [1]. These radii are available in the literature [2] and are important in considering structure models and breakup momentum distributions of such systems. The basis of these determinations is a comparison of calculated projectile-target total reaction cross sections, e.g. [3]

$\sigma_{R}(P)=2 \pi \int_{0}^{\infty} d b b\left[1-T_{P}(b)\right]=2 \pi \int_{0}^{\infty} d b b\left[1-\left|S_{P}(b)\right|^{2}\right]$,

with empirical interaction cross section data. Here $T_{P}(b)$, the square modulus of the Glauber elastic $S$-matrix for the projectile-target system, is the transparency of the two nuclei at impact parameter $b$.

Quantitative analyses of data for size determinations have used the optical limit [4] or static density (SD) approximation to Glauber theory, based on the geometric overlap of the projectile and target single particle densities. Radii deduced in this way, from data on a ${ }^{12} \mathrm{C}$ target at around $800 \mathrm{MeV} / \mathrm{A}$, also show little sensitivity to the precise radial form assumed for the ground state density, beyond its rms radius, leading some to speculate that the extracted values are, to a large extent, model independent. Recent work [5] would indicate however that such sensitivity depends strongly on the target mass.

While well studied and appropriate for 'regular' tightly bound mean field nuclei [6], such SD analyses are not expected to be reliable for very extended, correlated, few-body systems such as halo nuclei [7-9]. We review briefly the theoretical basis of the static density approximation and its relationship to Glauber theory for a general composite

${ }^{*}$ The financial support of the United Kingdom Engineering and Physical Sciences Research Council (EPSRC) in the form of Grants Nos. GR/J95867 and GR/K33026 is gratefully acknowledged. 
system. The quantitative implications of retaining explicitly these few-body effects are discussed.

\section{Glauber theory for composite systems}

When applied to a composite projectile, Glauber theory first makes the adiabatic approximation; i.e. the coordinates of each projectile constituent are frozen during the passage of the projectile through the interaction region with the target. At energies of order $800 \mathrm{MeV} / \mathrm{A}$ this approximation is certainly reliable. For an assumed $n$-body projectile, with ground state $\left|\Phi_{0}^{(n)}\right\rangle$, the composite projectile-target $S$-matrix is then

$S_{P}^{(n)}(b)=\left\langle\Phi_{0}^{(n)}\left|S_{1}\left(b_{1}\right) S_{2}\left(b_{2}\right) \ldots S_{n}\left(b_{n}\right)\right| \Phi_{0}^{(n)}\right\rangle$.

Here each $S_{j}\left(b_{j}\right)$ is the elastic $S$-matrix describing the scattering of projectile constituent $j$, independently, by the target and is a function of its own impact parameter $b_{j}$, Figure 1. It is clear that Eq. (2) represents the ground state matrix element of a many-body operator which will, in general, be sensitive to properties of the ground state wavefunction beyond its single particle density. Our interest is the level of this sensitivity for two- and threebody halo nuclear systems, the quantitative implications for their calculated cross section and hence for radii deduced by comparisons with data.

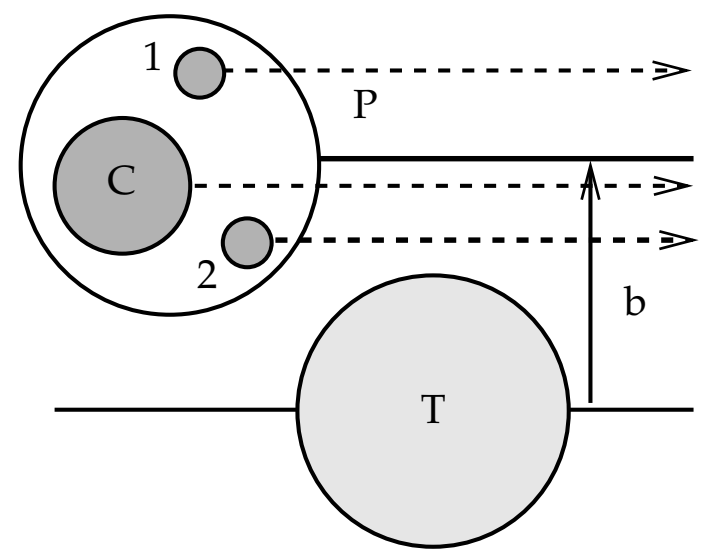

Figure 1. Glauber treatment of a threebody projectile-target collision at impact parameter $b$. The assumed straight line trajectories of each constituent at its own impact parameter is shown.

\subsection{Static density approximation}

In previous size analyses, the static density (SD) approximation [4] has been used to parameterise $S_{P}$. Here one neglects entirely the correlations between constituent nucleons in the projectile and target. For a mass $A$ projectile one therefore approximates

$\left|\Phi_{0}^{(A)}\left(\mathbf{r}_{1}, \mathbf{r}_{2}, \ldots, \mathbf{r}_{A}\right)\right|^{2} \rightarrow \prod_{j=1}^{A} \rho_{j}\left(\mathbf{r}_{j}\right)$

where, for all $j, \rho_{j}(\mathbf{r})=\rho_{P}(\mathbf{r}) / A$. That is each nucleon is assumed to move quite independently, but constrained by a ground state single particle density. The target is treated similarly.

In this limit the calculation of the reaction cross section can involve only the one-body densities $\rho_{P}$ and $\rho_{T}$ of the projectile and target. Specifically, the SD approximation to $S_{P}(b)$ is $[4,9]$

$S_{P}^{S D}(b)=\exp \left[i \mathcal{O}^{P T}(b)\right]$ 
Here $\mathcal{O}^{P T}(b)$ is determined by (double) folding the two densities with an assumed nucleonnucleon $(\mathrm{NN})$ formfactor, and the result then integrated along the straight line path of the projectile centre of mass at impact parameter $b$. With cylindrical coordinate decomposition $\mathbf{R}=\left(\mathbf{b}, R_{3}\right)$, then

$\mathcal{O}^{P T}(b)=\int_{-\infty}^{\infty} d R_{3} \int d \mathbf{r}_{\mathbf{1}} \int d \mathbf{r}_{\mathbf{2}} \rho_{P}\left(\left|\mathbf{r}_{1}\right|\right) \rho_{T}\left(\left|\mathbf{r}_{2}\right|\right) \hat{f}_{N N}\left(\left|\mathbf{R}+\mathbf{r}_{1}-\mathbf{r}_{2}\right|\right)$

Previous works [2] have usually assumed an absorptive zero-range NN amplitude, consistent with the optical theorem, in which case

$\hat{f}_{N N}(\mathbf{r})=\frac{i \bar{\sigma}_{N N}^{P T}}{2} \delta(\mathbf{r})$

For an isospin zero target, $\bar{\sigma}_{N N}^{P T}$ is then just the average of the free nn and np total cross sections at the energy of interest. A convenient parameterisation of these cross sections is given in [10].

\subsection{Few-body description}

We have presented the approximate SD approach in some detail for two reasons. The first is to clarify the nature of the approximations implicit in its use. The second is that we will use the SD approach to describe the localised nucleon- and core-target systems, i.e. to calculate the elastic $S$-matrix elements for the projectile constituents with the target; the $S_{j}\left(b_{j}\right)$ entering Eq. (2).

While inappropriate for the extended composite projectiles the SD approximation will be good for the nucleon- and core-target subsystems. Takigawa et al. [7] have demonstrated clearly the convergence of two-body and SD descriptions in the limit of tight valence nucleon binding. For composite halo systems we compare SD calculations with those which retain the essential few-body correlations by direct evaluation of Eq. (2) using realistic few-body wavefunctions [9]. Effective two-body $(n=2)$ and three-body $(n=3)$ systems are considered. The inputs to the calculation of the $S_{P}^{(n)}(b)$ and the cross section are now a few-body projectile wavefunction $\left|\Phi_{0}^{(n)}\right\rangle$ and the constituent-target $S$-matrices, calculated as above. These $S$-matrices are in fact highly constrained since they must be consistent with independent reaction cross section data for that subsystem; via Eq. (1) applied to the core- and nucleon-target systems.

This few-body picture presents a quite different description of the projectile-target interaction. Consider for example a three-body projectile of a pair of neutrons weakly bound to a more massive localised core. At an impact parameter at which the projectile density (circle in Figure 1) overlaps the target many spatial configurations of the constituents will not interact with the target. This figure represents the essential difference between the SD and few-body calculations. In the former, the neglect of spatial correlations implies an averaging over all constituent particle positions prior to a single scattering calculation of the resulting spherical density, Eqs. (3) and (4). In the few-body Glauber picture, the scattering must be calculated for each initial configuration of the constituents and then the resulting scattering amplitudes averaged over all configurations. Eq. (2) expresses this averaging over possible positions of the $n$-bodies, where each configuration must be weighted according to our best available many-body description of the systems involved. 


\section{Size determinations}

As was clarified above, the use of the approximate static density limit of Glauber theory suggests an explicit relationship between the projectile ground state density and calculated cross sections. This relationship has been exploited, due to the strong dependence of the calculated cross section on the root mean square radius of the density assumed, and is the basis of all earlier rms radius tabulations [2].

By contrast the few-body calculation, which proceeds via Eq. (2), involves the relative motion wavefunction of the few-body system and the constituent-target scattering $S$ matrices. The single particle density of the projectile does not appear explicitly and therefore the relationship of the calculated cross section and the projectile matter radius is far less obvious. To be definite we consider two- and three-body projectiles of masses $A+1$ or $A+2$ comprising a core $(C)$ and one $(1)$ or two $(1,2)$ point nucleons. There are therefore two sizes involved (i) that associated with the separation of the centres of mass of the constituents, and contained in $\left|\Phi_{0}^{(n)}\right\rangle$, and (ii) the size (the rms radius $\left\langle r^{2}\right\rangle_{A}^{1 / 2}$ ) of the $A$ particle core. The latter does not appear explicitly in the formalism although the spatial extent of the core will be implicit in the core-target $S$-matrix. A value for this core rms radius is nevertheless needed explicitly to compute the matter rms radius for composite projectiles.

For a three-body system, the size associated with the three-body separations is most conveniently expressed in terms of the rms value of the hyperradius variable $\rho^{2}=A r_{C}^{2}+$ $r_{1}^{2}+r_{2}^{2}$ [11], where the $r_{i}$ are the radial positions of the core and nucleons with respect to the $\mathrm{cm}$ of the composite. The relationship of this spatial extension, computed from the few-body wavefunction models, the core size, and the rms matter radius of the $A+2$ body system $\left\langle r^{2}\right\rangle_{A+2}^{1 / 2}$ then separates as

$(A+2)\left\langle r^{2}\right\rangle_{A+2}=A\left\langle r^{2}\right\rangle_{A}+\left\langle\rho^{2}\right\rangle$.

Similarly, for two-body systems

$$
(A+1)\left\langle r^{2}\right\rangle_{A+1}=A\left\langle r^{2}\right\rangle_{A}+\left\langle\rho^{2}\right\rangle
$$

where now $\rho^{2}=[A /(A+1)] r^{2}$ with $r$ the two-body separation. Where we quote rms radii for halo nuclei a core size will be assumed and specified. The comparison of fewbody calculations of cross sections with data essentially determines the spatial extensions $\left\langle\rho^{2}\right\rangle^{1 / 2}$ associated with the few-body wavefunctions, and only with additional input the projectile matter radii. For weakly bound systems these rms hyperradii are large and generate the dominant contributions to the radial relationships above.

\section{Calculations for two neutron halo systems}

We calculate reaction cross sections in the few-body approach for the two-neutron halo nuclei ${ }^{11} \mathrm{Li}$ and ${ }^{6} \mathrm{He}$. Comparisons of few-body and $\mathrm{SD}$ calculations for ${ }^{11} \mathrm{Li}$, the one-neutron halo nucleus ${ }^{11} \mathrm{Be}$, and the one-proton halo candidate ${ }^{8} \mathrm{~B}$ can also be found elsewhere [9]. We consider reactions on a ${ }^{12} \mathrm{C}$ target at $800 \mathrm{MeV} / \mathrm{A}$, dictated by our wish to connect the required $S$-matrices for the binary sub-systems with available cross section measurements. The target and all core nuclei are assumed to have Gaussian density 
distributions and the required nucleon- and core-target $S$-matrices are computed from Eqs. (3) and (4). The zero-range NN amplitude Eq. (5) is used unless stated otherwise. The ${ }^{12} \mathrm{C}$ rms radius used is $\left\langle r^{2}\right\rangle_{12}^{1 / 2}=2.32 \mathrm{fm}$.

\subsection{Calculations for ${ }^{11} \mathrm{Li}$}

Figure 2 shows the calculated ${ }^{11} \mathrm{Li}$ reaction cross sections in the few-body approach (solid line and full symbols) as a function of the rms hyperradius of the ${ }^{11} \mathrm{Li}$ wavefunction $\left\langle\rho^{2}\right\rangle_{11}^{1 / 2}$. The horizontal band shows the experimental interaction cross section datum $\sigma\left({ }^{11} \mathrm{Li}\right)=1060 \pm 10 \mathrm{mb}[12]$. From left to right the diamond symbols result from the P0 through P4 intruder 1s-wave (Faddeev) model wavefunctions of [13], with increasing $1 s$-state admixtures and halo radii. The extreme right hand point is a continuation of these model wavefunctions (P5) with $80 \%$ $\left(1 s_{1 / 2}\right)^{2}$ probability. The upright triangle is calculated using the L6A wavefunction [14], whose single particle density reproduces the measured cross section when using the SD approximation, see Figure 3. The inverted triangle uses the $0 s$-wave intruder wavefunction of Ref. [15].

To a good approximation the calculated cross sections scale linearly with the rms hyperradius, the experimental datum giving $\left\langle\rho^{2}\right\rangle_{11}^{1 / 2}=$ $9.46 \pm 0.25 \mathrm{fm}$. The ${ }^{11} \mathrm{Li}$ matter radius, assuming a ${ }^{9} \mathrm{Li}$ core radius $\left\langle r^{2}\right\rangle_{9}^{1 / 2}=2.30 \mathrm{fm}$, is therefore $\left\langle r^{2}\right\rangle_{11}^{1 / 2}=3.53 \pm 0.06 \mathrm{fm}$, to be compared with the SD deduced value of order $3.10 \mathrm{fm}$ ([2] and Figure 3). A comparison of the calculations of Figure 2, reexpressed in terms of the projectile rms matter radius, with SD calculations using the single particle densities derived from the same wavefunction models and core density, are shown in Figure 3. The solid points and line are the few-body calculations of Figure 2. The open symbols and dashed line are the SD results. The deduced radii from the two approaches, given by the intercepts with the empirical cross section, are as were noted above.

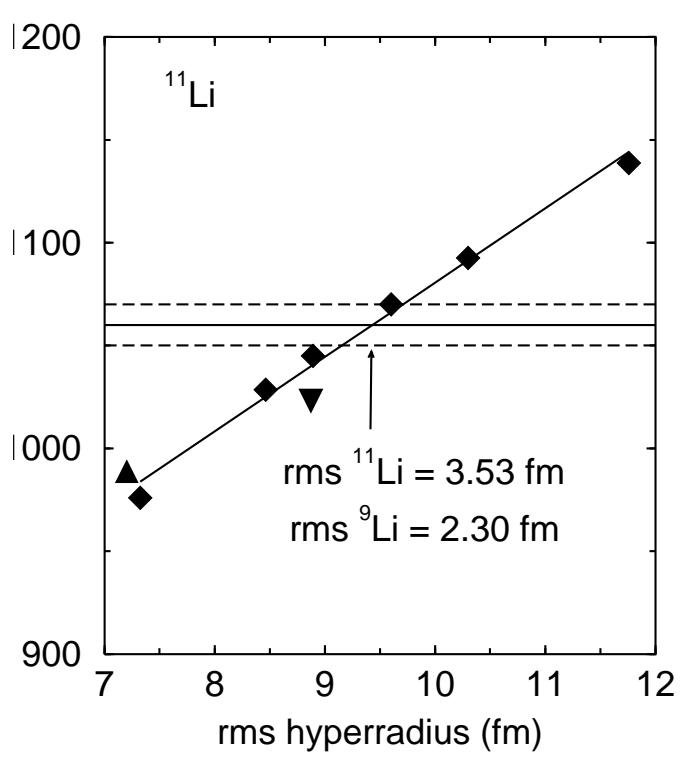

Figure 2. Calculated ${ }^{11} \mathrm{Li}+{ }^{12} \mathrm{C}$ reaction cross sections at $800 \mathrm{MeV} / \mathrm{A}$ for model Faddeev wavefunctions for ${ }^{11} \mathrm{Li}$ as a function of rms hyperradii.

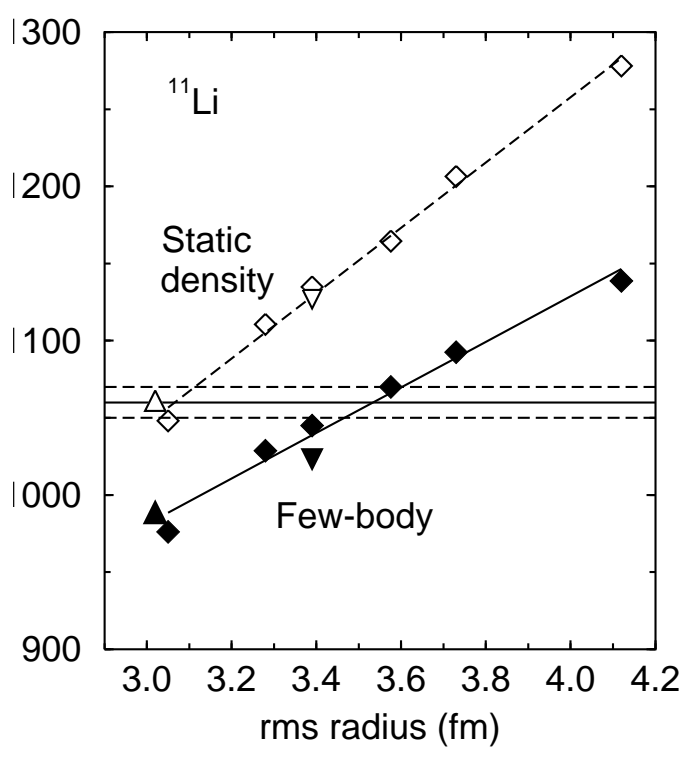

Figure 3. Calculated ${ }^{11} \mathrm{Li}+{ }^{12} \mathrm{C}$ reaction cross sections at $800 \mathrm{MeV} / \mathrm{A}$ in $\mathrm{SD}$ and few-body approaches as a function of the ${ }^{11} \mathrm{Li}$ rms radius. 


\subsection{Calculations for ${ }^{6} \mathrm{He}$}

Figure 4 shows few-body calculations for the ${ }^{6} \mathrm{He}+{ }^{12} \mathrm{C}$ system, also at $800 \mathrm{MeV} / \mathrm{A}$. The horizontal band shows the reported interaction cross section datum $\sigma\left({ }^{6} \mathrm{He}\right)=722 \pm 5 \mathrm{mb}$ [16].

The (lower) diamond symbols show the zero-range few-body calculations. From left to right these correspond to a series of Faddeev wavefunction models (P1, GB3, FC, FC6, $\mathrm{FB}, \mathrm{K}$ and $\mathrm{C}$ of [17]) in order of increasing rms hyperradius. The experimental datum gives $\left\langle\rho^{2}\right\rangle_{6}^{1 / 2}=5.85 \pm 0.10 \mathrm{fm}$, or, assuming a core radius $\left\langle r^{2}\right\rangle_{4}^{1 / 2}=1.50 \mathrm{fm}$, a ${ }^{6} \mathrm{He}$ matter radius of $\left\langle r^{2}\right\rangle_{6}^{1 / 2}=2.68 \pm 0.04 \mathrm{fm}$; larger than $\mathrm{SD}$ estimates which are of order 2.38 fm. For ${ }^{11} \mathrm{Li}, 1 s$-wave intruder models generate larger halo radii in a natural way. The configuration of the two neutrons in ${ }^{6} \mathrm{He}$ is however essentially pure $\left(p_{3 / 2}\right)^{2}$, and the longer ranged wavefunctions appearing in Figure 3, for example model $\mathrm{K}$ with rms hyperradius $5.8 \mathrm{fm}$, whose zero range cross section falls within the empirical cross section error bar, are larger because they are underbound compared to the empirical binding of $0.97 \mathrm{MeV}$.

To investigate this discrepancy we consider the role of the finite range of the NN formfactor in the nucleon- and core-target $S$-matrix calculations, Eqs. (3) and (4). These are expected to be of most significance in this the lightest of halo systems.

We consider two finite range prescriptions to replace Eq. (5). In the first we simply replace the $\delta$ function by a normalised Gaussian formfactor of range $0.65 \mathrm{fm}$, retaining the pure imaginary free NN cross section strength. In the second we incorporate fully the Gaussian NN formfactors of Ray (Table I of [18]) which have different ranges and strengths for the nn and np channels and which include a real component of the $\mathrm{NN}$ formfactor. In these calculations the rms radius of the ${ }^{12} \mathrm{C}$ target is held fixed at $2.32 \mathrm{fm}$, as used in the zero-range calculations. The radius of the ${ }^{4} \mathrm{He}$ density, entering the calculation of the core-target S-matrix, must however be adjusted to ensure the calculated $S$-matrix is consistent with the empirical ${ }^{4} \mathrm{He}+{ }^{12} \mathrm{C}$ cross section. The effect upon the calculated ${ }^{6} \mathrm{He}$ reaction cross sections are shown by the square and circular symbols, respectively, in Figure 4, each calculated for a subset of the Faddeev three-body models. The effects are

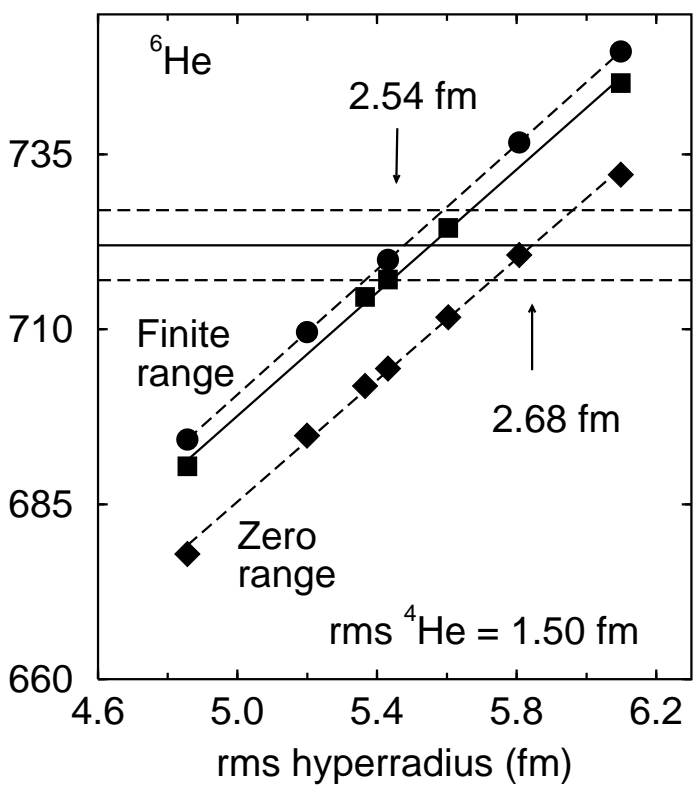

Figure 4. Calculated ${ }^{6} \mathrm{He}+{ }^{12} \mathrm{C}$ reaction cross sections at $800 \mathrm{MeV} / \mathrm{A}$ for model Faddeev wavefunctions for ${ }^{6} \mathrm{He}$ as a function of rms hyperradii. The symbols are described in the text. quite significant for this system.

The experimental datum now gives $\left\langle\rho^{2}\right\rangle_{6}^{1 / 2}=5.45 \pm 0.10 \mathrm{fm}$, or $\left\langle r^{2}\right\rangle_{6}^{1 / 2}=2.54 \pm 0.04$ $\mathrm{fm}$, consistent with those wavefunction models (FC, FC6) with the correct three-body binding energy. 


\section{Discussion and conclusions}

We have presented Glauber theoretical calculations of reaction cross sections for weakly bound two-neutron-halo nuclei at $800 \mathrm{MeV} / \mathrm{A}$ which treat explicitly the few-body character of these projectiles. Realistic Faddeev three-body wavefunction models are used for the ${ }^{11} \mathrm{Li}$ and ${ }^{6} \mathrm{He}$ systems. Treating these few-body degrees of freedom explicitly is shown to reduce calculated reaction cross sections, a consequence of an increased transparency of the collision at large impact parameters. It follows that matter radii, deduced by comparisons of calculated reaction cross sections with experimental data, are changed significantly from previously tabulated values.

We have clarified the way in which the separations of the three constituent bodies and the size of the core nucleus enter the calculation. We show that in three-body systems comparisons with data determine principally the rms hyperradius of the structure and less directly the halo nucleus matter radius.

An important feature of these more microscopic few-body calculations is that they compute the elastic $S$-matrix and the reaction cross section for the halo nucleus in terms of its few-body wavefunction and information obtained from the scattering of the constituent valence nucleons and core nucleus. This dependence on data for the constituents raises two difficulties which do not arise in the naive static density approach. Firstly, while interaction cross section data for the core and halo nuclei are now measured to high accuracy in the same fragmentation/transmission experiments, the available data for the nucleon-target systems are, by comparison, of poor quality. This lack of high precision cross section data for the nucleon, against which to assess the calculated nucleon-target $S$-matrices, now poses a significant uncertainty given the exceptional accuracy quoted on much of the core and halo nucleus data. The second uncertainty concerns ambiguities between the calculated (reaction) and measured (interaction) cross sections for the coretarget systems. While on experimental and theoretical grounds these cross sections are expected to be essentially equal for the fragile halo nuclei, possible differences between these measures for the more robust core nuclei with bound excited states is at present unclear. It will be extremely important to clarify this ambiguity experimentally and/or theoretically for selected systems.

While there is currently uncertainty in the precise structure of ${ }^{11} \mathrm{Li}$, with many available theoretical structures, the ${ }^{6} \mathrm{He}$ system provides a benchmark and is an essentially pure $\left(p_{3 / 2}\right)^{2}$ configuration. We have shown that few-body calculations which assume a zero-range nucleon-nucleon formfactor derive radii in excess of those of Faddeev models with the correct three particle separation energy. Effects of the finite range of the NN formfactor are shown to be significant for ${ }^{6} \mathrm{He}$, and appear to be needed to bring consistency between structure models and the interaction cross section data. It is considered extremely important that the $A=6$ systems, ${ }^{6} \mathrm{He}$ and ${ }^{6} \mathrm{Li}$, be studied very precisely, including those effects discussed above, to validate theoretical procedures in systems where three-body models are reliable and independent size measurements are available. Our deduced rms radius for ${ }^{11} \mathrm{Li},\left\langle r^{2}\right\rangle_{11}^{1 / 2}=3.53 \pm 0.06 \mathrm{fm}$, is consistent with wavefunctions with a significant $1 s$-state component.

The authors gratefully acknowledge the contribution of Dr Ian Thompson to this analysis through the provision of three-body wavefunctions for the ${ }^{11} \mathrm{Li}$ and ${ }^{6} \mathrm{He}$ systems in 
tabular form.

\section{REFERENCES}

1. I. Tanihata et al., Phys. Rev. Letts. 55, (1985) 2676; Phys. Lett. B160, (1985) 380.

2. I. Tanihata et al., Phys. Lett. B206, (1988) 592.

3. R.J. Glauber, in Lectures in Theoretical Physics, ed. W.E. Brittin (Interscience, N.Y., 1959) Vol. 1, p. 315.

4. W. Czyz and L.C. Maximon, Ann. Phys. (N.Y.) 52, (1969) 59.

5. M.Bush et al., Phys. Rev. C 53, (1996) 3009.

6. S. Kox et al., Phys. Rev. C 35, (1987) 1678.

7. N. Takigawa et al., Phys. Lett. B288, (1992) 244.

8. K. Yabana, Y. Ogawa and Y. Suzuki, Phys. Rev. C 45, (1992) 2909; Nucl. Phys. A539, (1992) 295.

9. J.S. Al-Khalili and J.A. Tostevin, Phys. Rev. Letts. 76, (1996) 3903.

10. S.K. Charagi and S.K. Gupta, Phys. Rev. C 41, (1990) 1610.

11. M.V. Zhukov and B. Jonson, Nucl. Phys. A589, (1995) 1.

12. T. Kobayashi et al., Phys. Lett. B232, (1989) 51.

13. I.J. Thompson and M.V. Zhukov, Phys. Rev. C 49, (1994) 1904.

14. J.M. Bang and I.J. Thompson, Phys. Lett. B270, (1992) 201.

15. L. Johannsen et al., Phys. Lett. B244, (1990) 357.

16. I. Tanihata et al., Phys. Lett. B289, (1992) 261.

17. J.S. Al-Khalili, J.A. Tostevin and I.J. Thompson, 'Radii of halo nuclei from cross section measurements', submitted to Phys. Rev. C.

18. L. Ray, Phys. Rev. C 20, (1979) 1857. 